[Contact e-mail: Olga.Lalakulich@theo.physik.uni-giessen.de]

\title{
Quark-hadron duality in lepton scattering off nuclei
}

\author{
Olga Lalakulich $*$ Natalie Jachowicz, Christophe Praet, and Jan Ryckebusch \\ Department of Subatomic and Radiation Physics, Ghent University, Belgium
}

\begin{abstract}
A phenomenological study of quark-hadron duality in electron and neutrino scattering on nuclei is performed. We compute the structure functions $F_{2}$ and $x F_{3}$ in the resonance region within a framework that includes the Dortmund-group model for the production of the first four lowest-lying baryonic resonances and a relativistic mean-field model for nuclei. We consider four-momentum transfers between 0.2 and $2.5 \mathrm{GeV}^{2}$. The results indicate that nuclear effects play a different role in the resonance and DIS region. We find that global but not local duality works well. In the studied range of four-momentum transfers, the integrated strength of the computed nuclear structure functions in the resonance region, is considerably lower than the DIS one.
\end{abstract}

\section{INTRODUCTION}

Nearly forty years ago, Bloom and Gilman found [1] that in electron scattering on protons the inclusive structure function $F_{2}$ in the resonance region oscillates around the DIS scaling curve and, after averaging, closely resembles it. This phenomenon is one of the ways quark-hadron duality reveals itself in physical processes. Generally quarkhadron duality establishes a relationship between the quark-gluon description of a certain phenomenon, which is theoretically justified in the DIS region, and the hadronic description, which is more convenient at medium and low energies. Understanding duality is also essential when establishing relationships between exclusive and inclusive processes. For a recent and detailed review of duality we refer the reader to Ref. [2].

So far, most theoretical studies of quark-hadron duality in lepton scattering were dealing with nucleon targets. The topic becomes of great practical interest when turning to nuclear targets and neutrino sources. The current precision measurements of the oscillation parameters require an efficient and accurate description of the neutrino-nucleus cross sections. Of particular interest is the resonance region and the possibility of linking it with the DIS region. A hadronic description of the neutrino-nucleus cross sections at low $Q^{2}$ requires the vector and axial transition form factors for each resonance. For the majority of the resonances, these transition form factors are not well constrained. Provided that one can establish that quark-hadron duality holds with a reasonable accuracy, one could think of using the DIS results for estimating the neutrino-nucleus cross sections in the resonance region. In that respect it is worth mentioning that in nuclei, the Fermi motion of the nucleons smears the observables, so that the averaging in the resonance region required for duality, proceeds to a certain extent automatically. The issue whether quark-hadron duality holds with sufficient accuracy in lepton-nucleus scattering, requires further theoretical and experimental investigation. The present paper addresses this issue from the theoretical point of view.

\footnotetext{
${ }^{*}$ Current affiliation: Institute for Theoretical Physics, Giessen University, Germanyff
} 
Recent electron scattering measurements at Jefferson Laboratory (JLab) have confirmed the validity of BloomGilman duality for the proton, deuterium [3] and iron [4] structure functions. Further experimental efforts are required for neutrino scattering. Among the upcoming neutrino experiments, Minerva [5, 6, 7] and SciBooNE[ 8, 9, 10] aim at measurements with carbon, iron and lead nuclei as targets. From the theoretical side, recent investigations of the phenomenon of duality for electron and neutrino scattering on nucleons include the works reported in Refs. [11, 12, 13]. These studies differ in the way they treat the resonant contributions and the way they parameterize the DIS structure functions. This paper extends the study of Ref. [13] about the duality phenomenon in the nucleon to nuclei.

For a free nucleon target, the structure functions generally depend on the transferred energy $\nu=E-E^{\prime}$ and four-momentum $Q^{2}=-q_{\mu} q^{\mu}$. At low $Q^{2}$ the $\nu$-distributions reveal several peaks, which correspond to various baryon resonances. We briefly sketch our theoretical approach to resonance production in nuclei in Section structure functions are defined in Section $\amalg$, At high $Q^{2}$ the structure functions exhibit scaling behavior, which is discussed in Section 【IC. Comparing the structure functions in these two regions allows one to check the basic features of duality and compare its validity for different targets and incoming leptons. Our results are presented in Section [II for electrons and Section [IV for neutrinos. Conclusions are given in Section $\nabla$

\section{FORMALISM}

We consider inclusive charged-current (CC) neutrino scattering from nuclei and its electromagnetic counterpart

$$
\nu_{l}\left(k^{\mu}\right)+A \rightarrow l^{-}\left(k^{\prime \mu}\right)+X, \quad l^{-}\left(k^{\mu}\right)+A \rightarrow l^{-}\left(k^{\prime \mu}\right)+X
$$

where $l$ is the lepton flavor, $A$ represents a nucleus with mass number $A$, and $k^{\mu}=(E, \vec{k})$ and $k^{\prime \mu}=\left(E^{\prime}, \vec{k}^{\prime}\right)$ are the four-momenta of the incoming and outgoing lepton respectively. We work in the laboratory frame of reference. The coordinate system is chosen such that the $z$-axis lies along the direction of the virtual photon, so that the transferred momentum is given by $q^{\mu}=k^{\mu}-k^{\prime \mu}=\left(\nu, 0,0, q^{z}\right)$. The lepton scattering proceeds in the $x z$-plane. In this section, we investigate the structure functions $F_{2}, 2 x F_{1}$ and $x F_{3}$, the latter being nonzero for neutrino reactions only. To this end, CP-violation effects are neglected for the case of electron scattering.

\section{A. Resonance production on a nucleus}

For lepton-nucleus scattering we describe the struck nucleus as a collection of bound nucleons. Assuming an independent-particle shell model, each nucleon occupies a nuclear shell $\alpha$ with a characteristic binding energy $e_{\alpha}$ and is described by the bound-state spinor $u_{\alpha}$. In the impulse approximation, an impinging lepton interacts with a single bound nucleon. Hence, the nuclear cross section can be expressed as an incoherent sum over all nucleons of one-nucleon cross sections weighted with the corresponding nucleon momentum distributions $n_{\alpha}$. For example, for a carbon nucleus, one has

$$
\begin{aligned}
\frac{d \sigma^{12}{ }^{12} C}{d Q^{2} d \nu}=\int d^{3} p[ & 2 \frac{\left.d \sigma_{\nu p}\right|_{1 s^{1 / 2}}}{d Q^{2} d \nu} n_{1 s^{1 / 2}}^{(p)}(|\vec{p}|)+4 \frac{\left.d \sigma_{\nu p}\right|_{1 p^{3 / 2}}}{d Q^{2} d \nu} n_{1 p^{3 / 2}}^{(p)}(|\vec{p}|) \\
& \left.+2 \frac{\left.d \sigma_{\nu n}\right|_{1 s^{1 / 2}}}{d Q^{2} d \nu} n_{1 s^{1 / 2}}^{(n)}(|\vec{p}|)+4 \frac{\left.d \sigma_{\nu n}\right|_{1 p^{3 / 2}}}{d Q^{2} d \nu} n_{1 p^{3 / 2}}^{(n)}(|\vec{p}|)\right] .
\end{aligned}
$$


TABLE I: Binding energies $(\mathrm{MeV})$ for carbon and iron nuclei

$\begin{array}{ccc} & \text { proton } & \text { neutron } \\ { }^{12} \mathrm{C}: & & \\ 1 s^{1 / 2} & 47.76 & 51.17 \\ 1 p^{3 / 2} & 16.76 & 19.87 \\ { }^{56} \mathrm{Fe}: & & \\ 1 s^{1 / 2} & 57.19 & 63.66 \\ 1 p^{3 / 2} & 43.11 & 50.12 \\ 1 p^{1 / 2} & 39.32 & 46.00 \\ 1 d^{5 / 2} & 27.64 & 34.84 \\ 2 s^{1 / 2} & 17.77 & 24.41 \\ 1 d^{3 / 2} & 16.55 & 23.01 \\ 1 f^{7 / 2} & 12.11 & 19.17 \\ 2 p^{3 / 2} & - & 5.99\end{array}$

This allows us to employ the one-body lepton-nucleon vertex that can be well constrained in experiments with a proton and deuteron target. The four-momentum of the bound nucleon can be written as $p^{\mu}=\left(m_{N}-e_{\alpha}, \vec{p}\right)$. Both the bound-state spinor $u_{\alpha}(\vec{p})$ and the corresponding binding energies are computed in the Hartree approximation

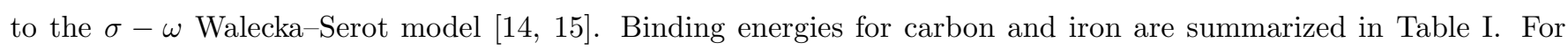
each shell, the nucleon momentum distribution $n_{\alpha}(|\vec{p}|)$ is constructed from the bound-state spinors, the normalization convention being

$$
\int d^{3} p n_{\alpha}(|\vec{p}|)=1
$$

These $n_{\alpha}(|\vec{p}|)$ are shown in Fig. 1, for the case of a carbon nucleus. Clearly, for a specific shell, the proton and neutron distributions are almost identical.

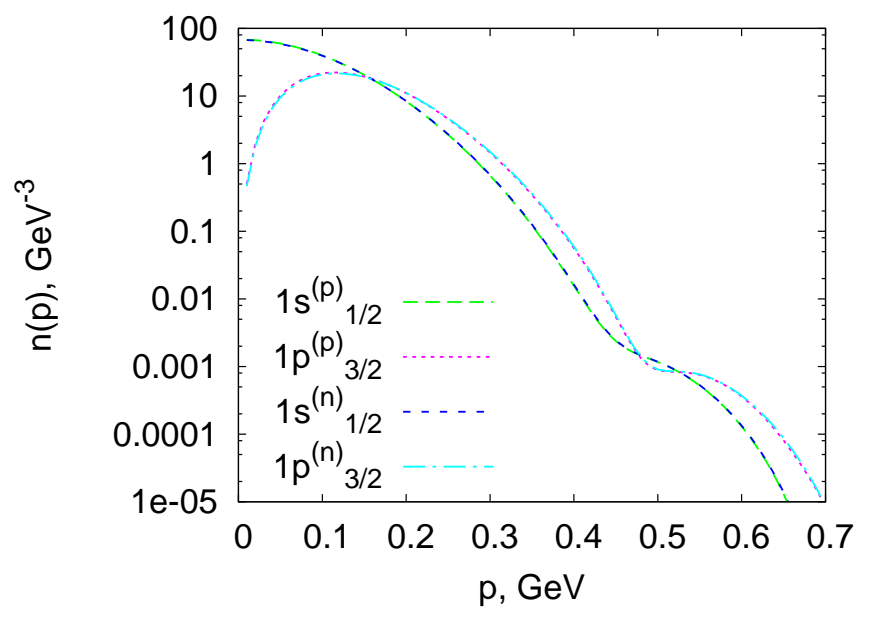

FIG. 1: Momentum distributions for proton and neutron shells in carbon.

After the interaction takes place inside the nucleus, the reaction products can escape the nucleus without interactions 
or they can undergo elastic and/or inelastic rescatterings with the other nucleons. Thus, the reaction strength is redistributed between different channels. All these processes are called the final state interactions (FSI). The effect of FSI can be large for a specific exclusive process, for example for quasi-elastic nucleon knockout [16], where the cross section can be suppressed by a factor of 2 . In one-pion production, the outgoing pion can be absorbed in the nucleus and thus mimic a quasi-elastic event. For a duality study, however, it suffices to consider inclusive reactions. Consequently, since the outgoing hadrons and the residual nucleus are not detected, we can make the assumption, following Ref. [17], that FSI can be disregarded.

Recently, duality in lepton-nucleon scattering was investigated theoretically within the Sato-Lee [18], Rein-Sehgal [19] and Dortmund-group [20] models for resonance production. In this paper, we follow the approach used in [20] and extend it to calculate the nuclear structure functions. In particular, in the resonance region we take into account the first four low-mass baryon resonances $P_{33}(1232), P_{11}(1440), D_{13}(1520), S_{11}(1535)$ and describe the vertices of their leptoproduction within a phenomenological form-factor approach. The nucleon structure functions $\mathcal{W}_{i}$ are defined by the standard expansion of the hadronic tensor

$$
W_{\mu \nu}=-g_{\mu \nu} \mathcal{W}_{1}+\frac{p_{\mu} p_{\nu}}{m_{N}^{2}} \mathcal{W}_{2}-i \varepsilon_{\mu \nu \lambda \sigma} \frac{p^{\lambda} q^{\sigma}}{2 m_{N}^{2}} \mathcal{W}_{3}+\frac{q_{\mu} q_{\nu}}{m_{N}^{2}} \mathcal{W}_{4}+\frac{p_{\mu} q_{\nu}+p_{\nu} q_{\mu}}{m_{N}^{2}} \mathcal{W}_{5}
$$

Each $\mathcal{W}_{i}$ depends on two independent kinematic variables, for example $Q^{2}$ and $\nu$, which are determined exclusively by the lepton kinematics. Another set of variables, namely $Q^{2}$ and $W$, is also possible, since the invariant mass $W$, defined as $W^{2}=(p+q)^{2}$, for a free target nucleon can be uniquely related to $Q^{2}$ and $\nu$ : $W^{2}=m_{N}^{2}+2 m_{N} \nu-Q^{2}$. The analytical expressions for the one-nucleon structure functions $F_{1}=m_{N} \mathcal{W}_{1}, F_{2}=\nu \mathcal{W}_{2}, F_{3}=\nu \mathcal{W}_{3}$ in terms of form factors for a free nucleon as well as the form factors themselves are given in [20]. The Fermi motion of the bound nucleon modifies the expression for the scalar product $(q \cdot p)$, so that the invariant mass $W^{2}=(p+q)^{2}$ will now depend on the nucleon momentum and binding energy. The variables $Q^{2}$ and $\nu$, being determined by lepton kinematics only, remain unaffected. Strictly speaking, the expansion in Eq. (2) is only valid for a free (on-mass shell) target nucleon. For a bound nucleon, all inclusive observables depend not only on $\nu$ and $Q^{2}$, but also on an additional independent kinematical variable, which can be chosen to be $p_{\mu} p^{\mu}=p^{2}$. Here, we make the assumption (see [21] for a detailed discussion) that expression (2) can still be used to define the bound-nucleon structure functions, and recalculate them keeping the kinematical variable $p^{2}$ as an independent one. The results are given below for the $W_{2}$ and $W_{3}$ structure functions. Equating $p^{2}=m_{N}^{2}$, the free-nucleon results of [20] are easily reproduced. For the spin-3/2 resonances $\left(P_{33}(1232)\right.$ and $D_{13}(1520)$ in our case) one has

$$
\mathcal{W}_{i}\left(Q^{2}, \nu, p^{2}\right)=\frac{2}{3 m_{N}} V_{i}\left(Q^{2}, \nu, p^{2}\right) R\left(W, M_{R}\right)
$$

where $R\left(W, M_{R}\right)$ is the finite representation of the $\delta$-function $\delta\left(W^{2}-M_{R}^{2}\right)$, which gives the relativistic Breit-Wigner distribution:

$$
R\left(W, M_{R}\right)=\frac{M_{R} \Gamma_{R}}{\pi} \frac{1}{\left(W^{2}-M_{R}^{2}\right)^{2}+M_{R}^{2} \Gamma_{R}^{2}},
$$

and the $V_{i}$ are given below. The upper and lower signs are for the positive $\left(P_{33}(1232)\right)$ and negative $\left(D_{13}(1520)\right)$ parity resonances, respectively.

$$
V_{2}=\frac{\left(C_{3}^{V}\right)^{2}+\left(C_{3}^{A}\right)^{2}}{M_{R}^{2}} Q^{2}\left[q \cdot p+p^{2}+M_{R}^{2}\right]+\left(\frac{\left(C_{4}^{V}\right)^{2}}{m_{N}^{2}}+\frac{\left(C_{5}^{V}\right)^{2}\left(Q^{2}+M_{R}^{2}\right)}{m_{N}^{2} M_{R}^{2}}+\frac{2 C_{4}^{V} C_{5}^{V}}{m_{N}^{2}}\right) Q^{2}\left[q \cdot p+p^{2} \mp m_{N} M_{R}\right]
$$




$$
\begin{aligned}
+ & \frac{C_{3}^{V} C_{4}^{V}}{m_{N} M_{R}} Q^{2}\left[q \cdot p+p^{2}+M_{R}^{2} \mp 2 m_{N} M_{R}\right]+\frac{C_{3}^{A} C_{4}^{A}}{m_{N} M_{R}} Q^{2}\left[q \cdot p+p^{2}+M_{R}^{2} \pm 2 m_{N} M_{R}\right]+C_{3}^{A} C_{5}^{A} \frac{m_{N}}{M_{R}} Q^{2} \\
+ & \frac{C_{3}^{V} C_{5}^{V}}{m_{N} M_{R}} Q^{2}\left[q \cdot p+p^{2}+M_{R}^{2} \mp 2 m_{N} M_{R}+Q^{2}\right]+\left[\left(C_{5}^{A}\right)^{2} \frac{m_{N}^{2}}{M_{R}^{2}}+\frac{\left(C_{4}^{A}\right)^{2}}{m_{N}^{2}} Q^{2}\right]\left[q \cdot p+p^{2} \pm m_{N} M_{R}\right], \\
V_{3} & =2 \frac{C_{3}^{V} C_{3}^{A}}{M_{R}^{2}}\left[2\left(Q^{2}-q \cdot p\right)^{2}+M_{R}^{2}\left(3 Q^{2}-4 q \cdot p\right)\right]+2\left[\frac{C_{4}^{V} C_{4}^{A}}{m_{N}^{2}}\left(Q^{2}-q \cdot p\right)-C_{4}^{V} C_{5}^{A}\right]\left(Q^{2}-q \cdot p\right) \\
& +2 \frac{C_{5}^{V} C_{3}^{A} q \cdot p-C_{4}^{V} C_{3}^{A}\left(Q^{2}-q \cdot p\right)}{M_{R} m_{N}}\left[2 M_{R}^{2} \mp 2 m_{N} M_{R}+Q^{2}-q \cdot p\right]+2\left[C_{5}^{V} C_{5}^{A}-\frac{C_{5}^{V} C_{4}^{A}}{m_{N}^{2}}\left(Q^{2}-q \cdot p\right)\right] q \cdot p \\
& +2\left[C_{3}^{V} C_{5}^{A} \frac{m_{N}}{M_{R}}-\frac{C_{3}^{V} C_{4}^{A}}{M_{R} m_{N}}\left(Q^{2}-q \cdot p\right)\right]\left(2 M_{R}^{2} \pm 2 m_{N} M_{R}+Q^{2}-q \cdot p\right)
\end{aligned}
$$

For spin-1/2 resonances we have

$$
\mathcal{W}_{i}\left(Q^{2}, \nu, p^{2}\right)=\frac{1}{m_{N}} V_{i}\left(Q^{2}, \nu, p^{2}\right) R\left(W, M_{R}\right),
$$

where

$$
\begin{aligned}
& V_{2}=2 m_{N}^{2}\left[\frac{\left(g_{1}^{V}\right)^{2}}{\mu^{4}} Q^{4}+\frac{\left(g_{2}^{V}\right)^{2}}{\mu^{2}} Q^{2}+\left(g_{1}^{A}\right)^{2}\right], \\
& V_{3}=4 m_{N}^{2}\left[\frac{g_{1}^{V} g_{1}^{A}}{\mu^{2}} Q^{2}+\frac{g_{2}^{V} g_{1}^{A}}{\mu}\left(M_{R} \pm m_{N}\right)\right],
\end{aligned}
$$

and $\mu=m_{N}+M_{R}$. The upper and lower signs again correspond to positive $\left(P_{11}(1440)\right)$ and negative $\left(S_{11}(1535)\right)$ parity resonances, respectively. In the case of electroproduction, all axial form factors should be put equal to zero and the weak vector form factors should be replaced by the electromagnetic ones for proton or neutron, depending on the target nucleon. To make the article self-contained, we present the transition form factors for each resonance. Electromagnetic and weak vector form factors were determined in [20] by fitting the electroproduction data on helicity amplitudes in the region $Q^{2}<3 \mathrm{GeV}^{2}$. Recently, it was shown [22] that in order to satisfy the asymptotics for helicity amplitudes at $Q^{2} \rightarrow \infty$, as prescribed by perturbative QCD, the vector form factors should also exhibit a certain asymptotic $Q^{2}$ behavior. Therefore, we refitted the form factors according to this prescription. In the region $Q^{2} \leq 4 \mathrm{GeV}^{2}$, however, the difference between our new fit and the one performed in [20] falls within the accuracy of the experimentally extracted helicity amplitudes. To be on the safe side for higher $Q^{2}$ values, further attempts to improve the fits of the form factors (for example, in accordance to upcoming data on helicity amplitudes) will be done within the framework of the arguments presented in [22]. The axial form factors are the ones used in [13] for the "fast" fall--off case. Thus, we use the following form factors

$$
\begin{gathered}
P_{33}(1232): C_{3}^{(p)}=\frac{2.14 / D_{V}}{1+Q^{2} / 4 M_{V}^{2}}, \quad C_{4}^{(p)}=\frac{-1.56 / D_{V}}{\left(1+Q^{2} / 7.3 M_{V}^{2}\right)^{2}}, \quad C_{5}^{(p)}=\frac{0.83 / D_{V}}{\left(1+Q^{2} / 0.95 M_{V}^{2}\right)^{2}}, \\
C_{i}^{(n)}=C_{i}^{(p)}, \quad C_{i}^{V}=C_{i}^{(p)} \\
C_{3}^{A}=0, \quad C_{4}^{A}=-C_{5}^{A} / 4, \quad C_{5}^{A}=\frac{1.2 / D_{A}}{1+Q^{2} / 3 M_{A}^{2}}, \quad C_{6}^{A}=m_{N}^{2} \frac{C_{5}^{A}}{m_{\pi}^{2}+Q^{2}},
\end{gathered}
$$




$$
\begin{gathered}
P_{11}(1440): g_{1}^{(p)}=\frac{2.2 / D_{V}}{1+Q^{2} / 1.2 M_{V}^{2}}\left[1 .+0.97 \ln \left(1 .+\frac{Q^{2}}{1 \mathrm{GeV}^{2}}\right)\right], \quad g_{2}^{(p)}=\frac{-0.76 / D_{V}}{\left(1+Q^{2} / 43 M_{V}^{2}\right)^{2}}\left[1-2.08 \ln \left(1+\frac{Q^{2}}{1 \mathrm{GeV}^{2}}\right)\right] \\
g_{i}^{(n)}=-g_{i}^{(p)}, \quad g_{i}^{V}=g_{i}^{(n)}-g_{i}^{(p)}, \\
g_{1}^{A}=\frac{-0.51 / D_{A}}{1+Q^{2} / 3 M_{A}^{2}}, \quad g_{3}^{A}=\frac{\left(M_{R}+m_{N}\right) m_{N}}{Q^{2}+m_{\pi}^{2}} g_{1}^{A(P)}, \\
D_{13}(1520): C_{3}^{(p)}=\frac{2.95 / D_{V}}{1+Q^{2} / 8.0 M_{V}^{2}}, \quad C_{4}^{(p)}=\frac{-1.05 / D_{V}}{\left(1+Q^{2} / 17 M_{V}^{2}\right)^{2}}, \quad C_{5}^{(p)}=\frac{-0.48 / D_{V}}{\left(1+Q^{2} / 37 M_{V}^{2}\right)^{2}} . \\
C_{3}^{(n)}=\frac{-1.13 / D_{V}}{1+Q^{2} / 8.0 M_{V}^{2}}, \quad C_{4}^{(n)}=\frac{0.46 / D_{V}}{\left(1+Q^{2} / 17 M_{V}^{2}\right)^{2}}, \quad C_{5}^{(n)}=\frac{-0.17 / D_{V}}{\left(1+Q^{2} / 37 M_{V}^{2}\right)^{2}}, \\
C_{i}^{V}=C_{i}^{(n)}-C_{i}^{(p)}, \\
C_{3}^{A}=0, \quad C_{4}^{A}=0, \quad C_{5}^{A}=\frac{-2.1 / D_{A}}{1+Q^{2} / 3 M_{A}^{2}}, \quad C_{6}^{A}=m_{N}^{2} \frac{C_{5}^{A}}{m_{\pi}^{2}+Q^{2}}, \\
S_{11}(1535): g_{1}^{(p)}=\frac{1.87 / D_{V}}{1+Q^{2} / 1.2 M_{V}^{2}}\left[1+7.07 \ln \left(1+\frac{Q^{2}}{1 \mathrm{GeV}^{2}}\right)\right], \quad g_{2}^{(p)}=\frac{0.64 / D_{V}}{\left(1+Q^{2} / 17 M_{V}^{2}\right)^{2}}\left[1+1.0 \ln \left(1+\frac{Q^{2}}{1 \mathrm{GeV}^{2}}\right)\right] \\
g_{i}^{(n)}=-g_{i}^{(p)}, \quad g_{i}^{V}=g_{i}^{(n)}-g_{i}^{(p)}, \\
g_{1}^{A}=\frac{-0.21 / D_{A}}{1+Q^{2} / 3 M_{A}^{2}}, \quad g_{3}^{A}=\frac{\left(M_{R}-m_{N}\right) m_{N}}{Q^{2}+m_{\pi}^{2}} g_{1}^{A} .
\end{gathered}
$$

Here, $D_{V}=\left(1+Q^{2} / M_{V}^{2}\right)^{2}$ with $M_{V}=0.84 \mathrm{GeV}$ and $D_{A}=\left(1+Q^{2} / M_{A}^{2}\right)^{2}$ with $M_{A}=1.05 \mathrm{GeV}$. The weak form factors presented here are determined for the excitation of the $R^{+}$resonance state, i.e. for neutrino scattering on a neutron. For the excitation of the double charged states, which is possible for isospin-3/2 resonances in neutrinoproton scattering, the isospin relation gives an additional factor $\sqrt{3}$ for each form factor.

For the resonance widths we use the so called running widths $\Gamma_{R}(W)$, as they were presented in Ref. [20]:

$$
\Gamma_{R}(W)=\Gamma_{R}^{0}\left(\frac{p_{\pi}(W)}{p_{\pi}\left(M_{R}\right)}\right)^{2 s_{R}}
$$

where $s_{R}$ is the spin of the resonance, on-shell widths are $\Gamma_{\Delta}^{0}=0.12 \mathrm{GeV}, \Gamma_{P 1440}^{0}=0.350 \mathrm{GeV}, \Gamma_{D 1520}^{0}=0.125 \mathrm{GeV}$, $\Gamma_{S 1535}^{0}=0.150 \mathrm{GeV}$, and

$$
p_{\pi}(W)=\frac{1}{2 W} \sqrt{\left(W^{2}-m_{N}^{2}-m_{\pi}^{2}\right)^{2}-4 m_{N}^{2} m_{\pi}^{2}} .
$$

\section{B. Definition of the nuclear structure functions}

For nuclear targets, the nuclear structure functions $\mathcal{W}_{i}^{A}$ can be defined in the standard manner by means of the expansion of the nuclear hadronic tensor

$$
W_{\mu \nu}^{A}=-g_{\mu \nu} \mathcal{W}_{1}^{A}+\frac{p_{\mu}^{A} p_{\nu}^{A}}{M_{A}^{2}} \mathcal{W}_{2}^{A}-i \varepsilon_{\mu \nu \lambda \sigma} \frac{p_{A}^{\lambda} q^{\sigma}}{2 M_{A}^{2}} \mathcal{W}_{3}^{A}+\frac{q_{\mu} q_{\nu}}{M_{A}^{2}} \mathcal{W}_{4}^{A}+\frac{p_{\mu}^{A} q_{\nu}+p_{\nu}^{A} q_{\mu}}{M_{A}^{2}} \mathcal{W}_{5}^{A},
$$

where $p^{A, \mu}=\left(M_{A}, \overrightarrow{0}\right)$ is the four-momentum of the target nucleus with mass $M_{A}$ in the laboratory frame of reference.

In the impulse approximation we are dealing with the bound nucleon as a target, so we must relate the one-bound-nucleon structure functions introduced in the previous section to nuclear ones. We follow the prescription of Ref. [23] and express the nuclear structure functions in terms of the nucleon ones in terms of a convolution of the type

$$
W_{\mu \nu}^{A}=\sum_{\alpha} \int d^{3} p\left(2 j_{\alpha}+1\right) n_{\alpha}(p)\left(W_{\mu \nu(\alpha)}^{p}+W_{\mu \nu(\alpha)}^{n}\right),
$$


where $\alpha$ extends over single-particle shells in the target nucleus and $2 j_{\alpha}+1$ specifies their occupancies.

It is worth stressing that in the original paper [23] as well as in 21] an additional phase-space correction factor $E_{p} / m_{N}$ is introduced in the expression (14) to preserve the space volume under Lorentz transformation. Since we construct a momentum distribution from wave functions normalized as $u_{\alpha}^{\dagger} u_{\alpha}=1$ for each shell $\alpha$, our correction factor must be equal to 1 .

Substituting (2) and (13) in (14), one arrives at

$$
\begin{aligned}
& \mathcal{W}_{1}^{A}\left(Q^{2}, \nu\right)=\sum_{\alpha} \mathcal{W}_{1}^{(\alpha)}\left(Q^{2}, \nu\right)=\sum_{\alpha} \int d^{3} p\left(2 j_{\alpha}+1\right) n_{\alpha}(p)\left[\mathcal{W}_{1}\left(Q^{2}, \nu, p^{2}\right)+\mathcal{W}_{2}\left(Q^{2}, \nu, p^{2}\right) \frac{|\vec{p}|^{2}-p_{z}^{2}}{m_{N}^{2}}\right] \\
& \mathcal{W}_{2}^{A}\left(Q^{2}, \nu\right)=\sum_{\alpha} \mathcal{W}_{2}^{(\alpha)}\left(Q^{2}, \nu\right)=\sum_{\alpha} \int d^{3} p\left(2 j_{\alpha}+1\right) n_{\alpha}(p) \mathcal{W}_{2}\left(Q^{2}, \nu, p^{2}\right)\left[\frac{|\vec{p}|^{2}-p_{z}^{2}}{m_{N}^{2}} \frac{Q^{2}}{q_{z}^{2}}+\left(\frac{(p \cdot q)}{m_{N} \nu}\right)^{2}\left(1+\frac{p_{z}}{q_{z}} \frac{Q^{2}}{(p \cdot q)}\right)^{2}\right] .
\end{aligned}
$$

This prescription guarantees, that as $Q^{2}$ tends to zero, the longitudinal structure function $\mathcal{W}_{L}$ also tends to zero as expected for the real photon:

$$
\lim _{Q^{2} \rightarrow 0}\left[\frac{\nu^{2}}{Q^{2}} \mathcal{W}_{2}^{A}\left(Q^{2}, \nu\right)-\mathcal{W}_{1}^{A}\left(Q^{2}, \nu\right)\right]=0 .
$$

In neutrino experiments one can also measure the $\mathcal{W}_{3}$ structure function, for which our definition gives:

$$
\mathcal{W}_{3}^{A}\left(Q^{2}, \nu\right)=\sum_{\alpha} \int d^{3} p\left(2 j_{\alpha}+1\right) n_{\alpha}(p) \mathcal{W}_{3}\left(Q^{2}, \nu, p^{2}\right) \frac{M_{A}}{m_{N}^{2}} \frac{p^{0} q^{z}-\nu p^{z}}{q_{z}}
$$

Note that $\mathcal{W}_{3}$ depends on the nucleus mass $M_{A}$. Realizing that the Bjorken variable for a nucleus $\left(x_{A}=Q^{2} / 2 M_{A} \nu\right)$ differs from the one for a nucleon $\left(x=Q^{2} / 2 m_{N} \nu\right)$, the function that is independent of $M_{A}$ is $x_{A} F_{3}^{A}$ :

$$
x_{A} F_{3}^{A}=\sum_{\alpha} \int d^{3} p\left(2 j_{\alpha}+1\right) n_{\alpha}(p) x F_{3}\left(Q^{2}, \nu, p\right) \frac{1}{m_{N}} \frac{p^{0} q^{z}-\nu p^{z}}{q_{z}} .
$$

According to the definition (15), $F_{2}^{A}=\nu \mathcal{W}_{2}^{A}$ and $x_{A} F_{1}^{A}=x_{A} M_{A} \mathcal{W}_{1}^{A}$ are also independent on $M_{A}$.

Within the adopted approach there is no unambiguous recipe for deciding whether one should keep $m_{N}$ in the denominators of (15) or replace it with some effective mass, that corrects for the binding energy. For the numerical calculations presented here, we have opted to use the expression (15) and interpret the $m_{N}$ as the free nucleon mass.

The integration over $d^{3} p=|\vec{p}|^{2} d|\vec{p}| d \cos \gamma_{p} d \varphi_{p}$ in Eq. (15) is performed in the following way. Integration over the azimuthal angle $d \varphi_{p}$ gives $2 \pi$, since no structure function depends on it. The phase space in the plane determined by the absolute momentum value $|\vec{p}|$ and polar angle $\gamma_{p}$ is restricted by the condition $W^{2}>W_{\text {min }}^{2}$. For one-pion production one has that $W_{\min }=m_{N}+m_{\pi}$. For a a bound nucleon this condition translates into

$$
p_{0}^{2}-|\vec{p}|^{2}+2 p_{0} \nu-2|\vec{p}| \sqrt{Q^{2}+\nu^{2}} \cos \gamma_{p}-Q^{2}>W_{\text {min }}^{2} .
$$

When performing the $d^{3} p$ integrations, the above condition determines the boundaries of the absolute bound-nucleon momentum $|\vec{p}|$ for a given $\cos \gamma_{p}, Q^{2}$ and $\nu$ :

$$
|\vec{p}|_{ \pm}=-\sqrt{Q^{2}+\nu^{2}} \cos \gamma_{p} \pm \sqrt{\left(Q^{2}+\nu^{2}\right) \cos ^{2} \gamma_{p}+p_{0}^{2}+2 p_{0} \nu-Q^{2}-W_{m i n}^{2}}
$$

The sign of the quantity $W_{\min }^{2}+Q^{2}-p_{0}^{2}-2 p_{0} \nu$ discriminates between two classes of kinematic conditions. In what follows we provide a discussion of the values of $p_{\min }$ and $p_{\max }$ in the phase-space integration $\int_{p_{\min }}^{p_{\max }} d p$ for a positive and negative sign of $W_{\text {min }}^{2}+Q^{2}-p_{0}^{2}-2 p_{0} \nu$. For

$$
W_{m i n}^{2}+Q^{2}-p_{0}^{2}-2 p_{0} \nu<0
$$


the $|\vec{p}|_{-}$calculated according to (20) is negative, so one should take $p_{\min }\left(Q^{2}, \nu, \cos \gamma_{p}\right)=0$. This means that the phase space (21) is accessible for a nucleon with arbitrarily small three-momentum, including $|\vec{p}|=0$, as is the case for a free nucleon. When the condition (21) is fullfilled, $p_{\max }\left(Q^{2}, \nu, \cos \gamma_{p}\right)=|\vec{p}|_{+}\left(Q^{2}, \nu, \cos \gamma_{p}\right)$ for all polar angles $\gamma_{p}$

Increasing the phase space for the bound nucleon does not necessarily imply that the cross section grows, because each point in the phase space gets weighted with a momentum distribution of the type shown in Fig. 11 Cross sections and structure functions for high $|\vec{p}|$ are strongly suppressed and the major contributions stem from the momenta inside the Fermi sphere.

For

$$
W_{\text {min }}^{2}+Q^{2}-p_{0}^{2}-2 p_{0} \nu>0
$$

the $|\vec{p}|_{ \pm}$are only defined for backward moving target nucleons. The restrictions on $\cos \gamma_{p}$ for given $Q^{2}$ and $\nu$ come from the condition

$$
\left(Q^{2}+\nu^{2}\right) \cos ^{2} \gamma_{p}+p_{0}^{2}+2 p_{0} \nu-Q^{2}-W_{\text {min }}^{2}>0
$$

which gives

$$
-1<\cos \gamma_{p}\left(Q^{2}, \nu\right)<-\sqrt{\frac{W_{m i n}^{2}+Q^{2}-p_{0}^{2}-2 p_{0} \nu}{Q^{2}+\nu^{2}}}
$$

Since the minimal value of the three-momentum $|\vec{p}|_{-}$is positive in this case, the accessibility to this $\left(Q^{2}, \nu\right)$ region crucially depends on a nucleon already moving, which is only possible for a bound nucleon. This region of phase space grows in importance with increasing $Q^{2}$.

For $Q^{2}=0.1 \mathrm{GeV}^{2}$ and different $\nu$, the typical phase spaces available for a $1 s^{1 / 2}$ proton in carbon are shown in Fig. 2. We use polar coordinates for the variables $|\vec{p}|$ and $\gamma_{p}$. The left (right) panel corresponds with the condition (22) $(\underline{21})$. For each $Q^{2}$ and $\nu$, thick lines represent $|\vec{p}|_{+}$and thin lines $|\vec{p}|_{-}$. The points where the $|\vec{p}|_{+}$and $|\vec{p}|_{-}$ lines coincide correspond to the upper boundary on $\cos \gamma_{p}$, as calculated in Eq.(24). Remark that the available phase space in $\left(|\vec{p}|, \cos \gamma_{p}\right)$ is contained within a circle. At $\nu=0.4 \mathrm{GeV}$ the left part of the circle is not shown because it corresponds to values of $|\vec{p}|$ larger than $1 \mathrm{GeV}$. The momentum distribution of nucleons in nuclei will reduce those contributions to negligible proportions.

The phase space collapses to one point $\cos \gamma_{p}=-1,|\vec{p}|=\sqrt{Q^{2}+\nu_{\min }^{2}}$ for $\nu_{\min }=-p_{0}+W_{\min }$. Remark that for a bound nucleon the minimal value of $\nu$ does not depend on $Q^{2}$. Physically this means that for any $Q^{2}$ there is a bound nucleon moving backward fast enough to fulfill the requirement $(p+q)^{2}>W_{m i n}^{2}$. Thus, contrary to the free nucleon case, for the off-shell nucleon the pion production threshold is defined in terms of $\nu$ rather than invariant mass and, strictly speaking, is independent of $Q^{2}$.

At high $Q^{2}$, however, using $\nu_{\min }=-p_{0}+W_{\min }$ is not convenient for calculations, because all observables are strongly suppressed for large $\vec{p}$ inspite of the fact that the phase space is available. In our numerical calculations we have not considered nucleon momenta beyond three times the Fermi momentum. We stress that the phase space boundaries derived here depend on our assumption about the form of the four-momentum for the bound nucleon, which was taken as $p^{\mu}=\left(m_{N}-e_{\alpha}, \vec{p}\right)$. 

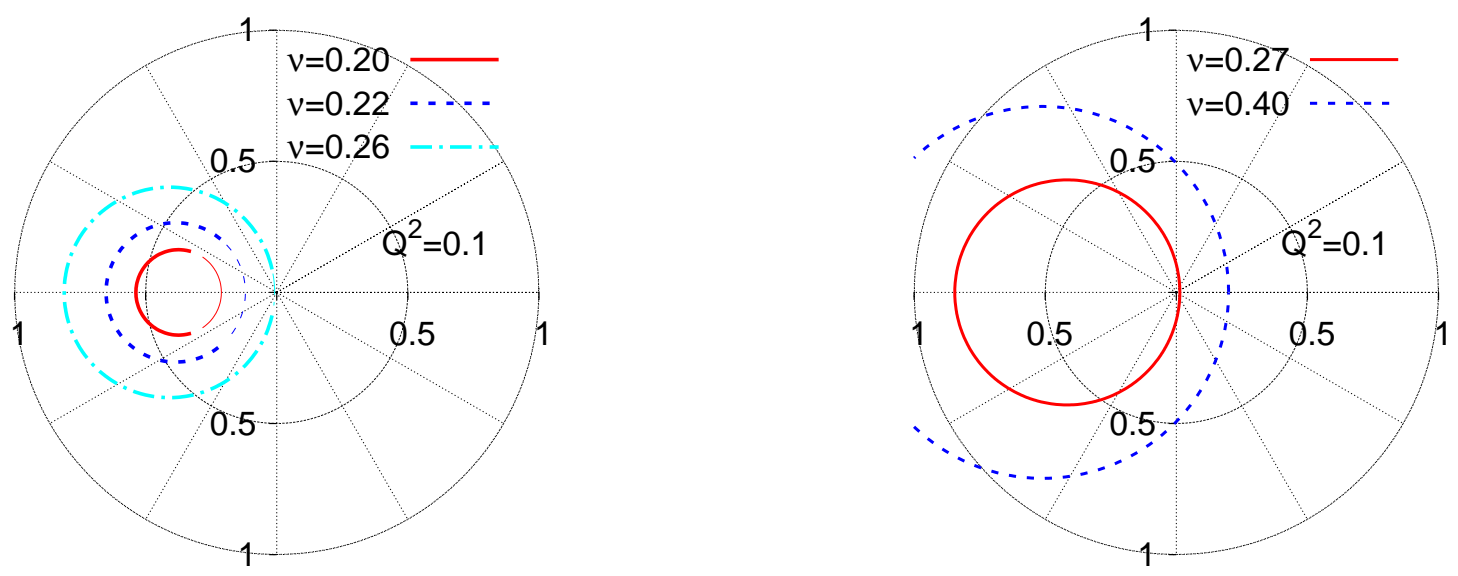

FIG. 2: Sketch of the available $\left(|\vec{p}|, \gamma_{p}\right)$ phase space in polar coordinates. We consider a $1 s^{1 / 2}$ proton in carbon for $Q^{2}=$ $0.1 \mathrm{GeV}^{2}$ and different $\nu$. The $|\vec{p}|$ is expressed in GeV. The left (right) panel corresponds with kinematics conditions obeying the condition of Eq. (22) (of Eq. (21)).

\section{DIS region and scaling variable}

In the kinematical regime of high $Q^{2}$ and $\nu$, the so-called Bjorken limit, the structure functions depend only on the Bjorken variable $x=Q^{2} / 2 m_{N} \nu$ when one neglects higher-twist effects. This phenomenon of no observed $Q^{2}$ dependence for a fixed $x$ value is called Bjorken scaling. At these energies, the lepton scattering on nucleons and nuclei is dominated by deep inelastic scattering with a multiple-particle hadronic final state. Deep inelastic scattering on nuclei was intensively studied experimentally since the sixties. This experimental information will be used as DIS input for our investigation.

For electron-carbon scattering, $F_{2}$ was measured by the BCDMS Collaboration [24, 25] for $30 \mathrm{GeV}^{2}<Q^{2}<$ $200 \mathrm{GeV}^{2}$. We choose several sets of data at different $Q^{2}=Q_{D I S}^{2}: 30,45$ and $50 \mathrm{GeV}^{2}$. As expected from Bjorken scaling, for most of the $x$ region the data coincide with an accuracy better than $5 \%$. For iron, the neutrino scattering results are available from the CCFR [26] and $\mathrm{NuTeV}$ [27] collaborations.

Scaling structure functions are conventionally plotted against the Bjorken variable $x$. Violation of Bjorken scaling comes from target-mass corrections and higher-twist effects. In the scaling region, the Nachtmann variable $\xi=$ $2 x /\left(1+\sqrt{1+4 m_{N}^{2} x^{2} / Q^{2}}\right)$ was shown [28, 29] to be a better alternative, because it implicitly includes the kinematical part of the target-mass correction, which can be important at large $x$ and low $Q^{2}$. Expanding the inverse of this variable in a power series of $1 / Q^{2}$, we recover the variable $1 / \xi \approx \omega^{\prime}=\left(2 m_{N} \nu+m_{N}^{2}\right) / Q^{2}$, used by Bloom and Gilman in their pioneering work on duality. For large $\nu$, one has $\omega^{\prime} \approx 1 / x$.

\section{DUALITY IN ELECTROPRODUCTION}

In the case of an isoscalar target nucleon, and for $Q^{2}>0.5 \mathrm{GeV}^{2}$, it was shown [13] that Bloom-Gilman duality holds at the level of $20 \%$. Here, we compute the nuclear structure functions $F_{2}^{A}$ and $x_{A} F_{1}^{A}$ along the model outlined in Section II. The results of our description of the resonance region in terms of hadronic degrees of freedom are then compared to DIS data. 
In Figure 3, the bound-nucleon structure functions $F_{2}$ and $2 x F_{1}$ for a proton in the $1 s^{1 / 2}$ and $1 p^{3 / 2}$ carbon shells are contrasted with the structure functions for a free proton. They are plotted versus the Nachtmann variable $\xi$ for $Q^{2}=0.2,0.85$, and $2.4 \mathrm{GeV}^{2}$, with the largest $Q^{2}$ curves covering the largest $\xi$ values. Similar to the free-nucleon case, for a fixed $Q^{2}$, the peak at larger $\xi$ corresponds to the $\Delta$ resonance and the peak at smaller $\xi$ corresponds to the second resonance region. One can easily notice the effect of smearing: the two resonance regions are distinguishable only at low $Q^{2}$. Fermi smearing proceeds differently for different shells, which in turn introduces an additional averaging when summing over shells. One can also observe that the bound-nucleon curves extend to higher $\xi$ values than the free nucleon ones. This additional contribution comes from the phase space at low $\nu$ values, which is shown in the left panel of Fig. 2 and discussed in Section IB At high $\xi$ the $F_{2}$ and $2 x F_{1}$ for the $1 s^{1 / 2}$ shell are significantly lower than for the $1 p^{3 / 2}$ shell. At high $\xi$ the phase space extends to relatively large bound-nucleon momenta. For those momenta the momentum distribution for a shell close to the Fermi surface (like $1 p^{3 / 2}$ in carbon) is larger than for a deep-lying shell (like $1 s^{1 / 2}$ in carbon).
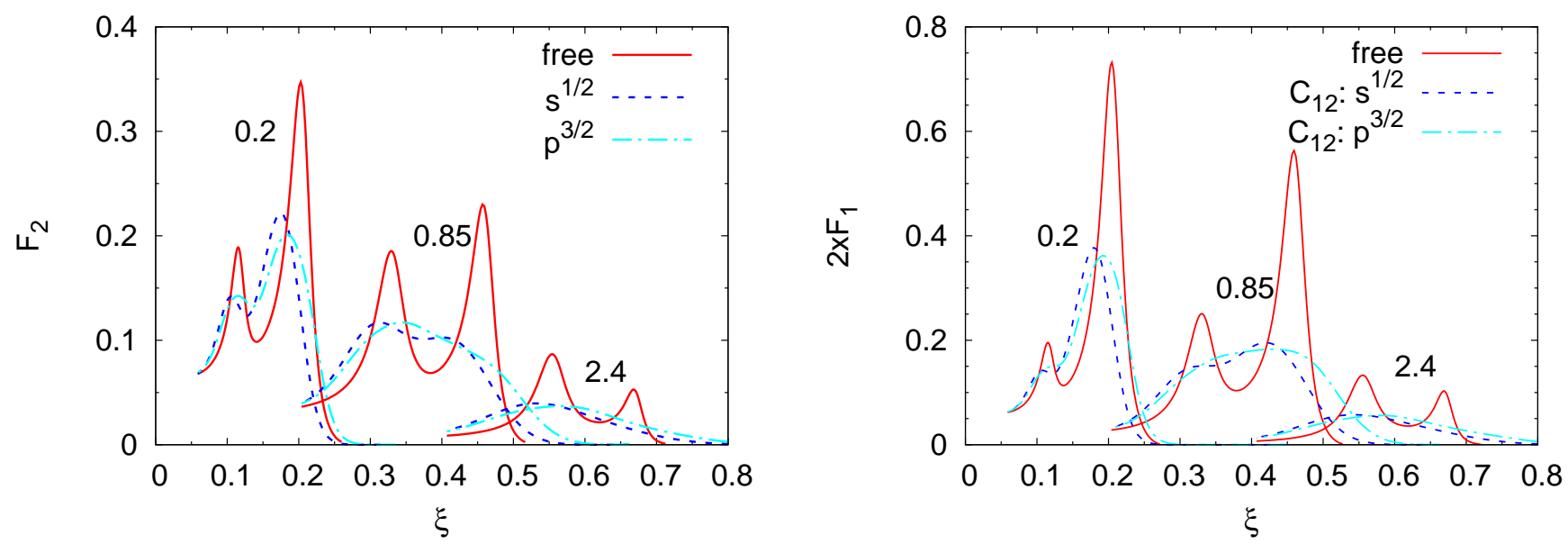

FIG. 3: (color online) Structure functions $F_{2}$ (left) and $2 x F_{1}$ (right) for a free proton (solid curve), for $1 s^{1 / 2}$ (dashed curve) and $1 p^{3 / 2}$ (dash-dotted curve) protons in ${ }^{12} \mathrm{C}$. The three sets of curves correspond to $Q^{2}=0.2,0.85$, and $2.4 \mathrm{GeV}^{2}$

Fig. 4 shows the carbon structure function per nucleon $F_{2}^{e^{12} C} / A$ in the resonance region for several $Q^{2}$ values, from 0.45 to $3.3 \mathrm{GeV}^{2}$. When investigating duality for a free nucleon, we took the average over free proton and neutron targets, thus considering the isoscalar structure function. Since the carbon nucleus contains an equal number of protons and neutrons, averaging over isospin is performed automatically. At $Q^{2}=0.45 \mathrm{GeV}^{2}$, the $\Delta$ peak is pronounced and can still be distinguished from the second resonance peak, which is also visible. At higher $Q^{2}$ one cannot distinguish the resonance structure anymore and the first and second resonance region merge into one broad peak.

In the left panel of Fig. 4 the resonance structure functions are compared with data obtained by the BCDMS Collaboration [24, 25] in muon-carbon scattering in the DIS region $\left(Q^{2} \sim 30-50 \mathrm{GeV}^{2}\right)$. They are shown as experimental points connected by smooth curves. For different $Q^{2}$ values, the curves agree within $5 \%$ in most of the $\xi$ region, as expected from Bjorken scaling. One observes that, as $Q^{2}$ increases, the resonance peaks decrease in height and slide along the DIS curve. This means that global duality holds for electron scattering on nuclei. To characterize 

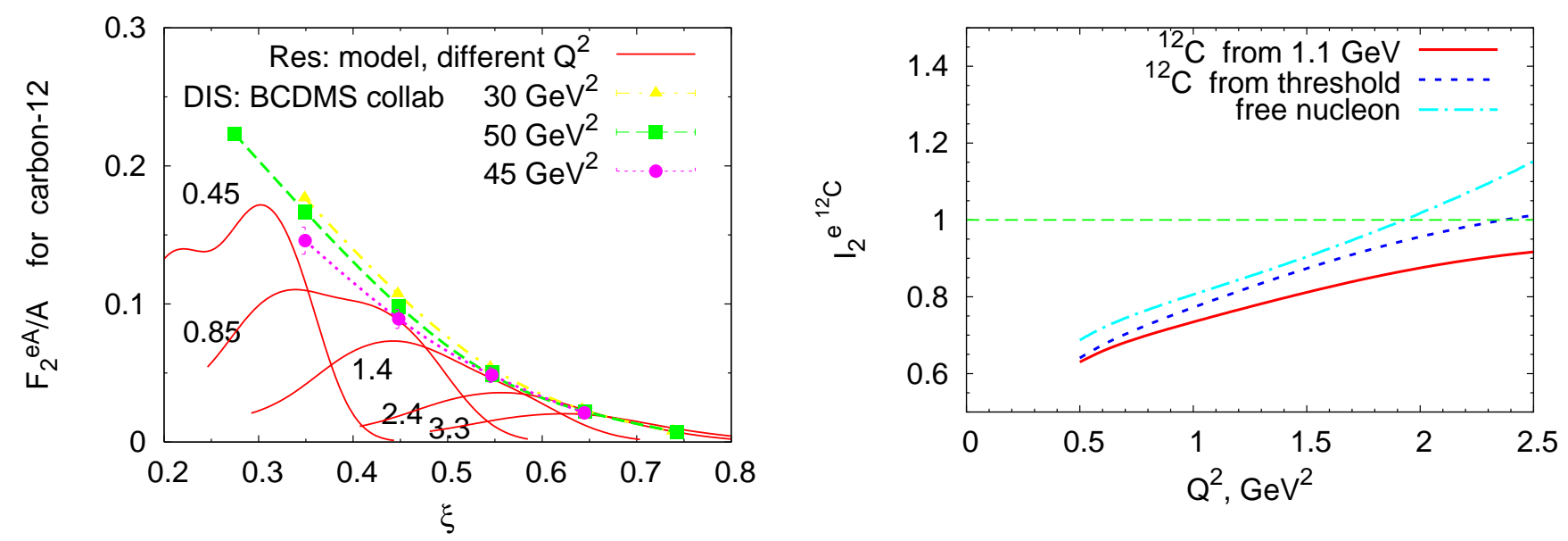

FIG. 4: (color online) Duality for the $F_{2}^{e^{12} C}$ structure function. (Left) Resonance curves $F_{2}^{e^{12} C} / 12$ as a function of $\xi$, for $Q^{2}=0.45,0.85,1.4,2.4$ and $3.3 \mathrm{GeV}^{2}$ (indicated on the spectra), compared with the experimental data 24, 25] in the DIS region at $Q_{D I S}^{2}=30,45$ and $50 \mathrm{GeV}^{2}$. (Right) Ratio $I_{2}$ defined in Eq. (25) for the free nucleon (dash-dotted line), and ${ }^{12}$ c. We consider the under limits determined by $\tilde{W}=1.1 \mathrm{GeV}$ (solid line) and by the threshold value (dotted line).

local duality, we consider the ratio of the integrals of the resonance (res) and DIS structure functions

$$
I_{i}\left(Q^{2}\right)=\frac{\int_{\xi_{\min }}^{\xi_{\max }} d \xi \mathcal{F}_{i}^{(\mathrm{res})}\left(\xi, Q^{2}\right)}{\int_{\xi_{\min }}^{\xi_{\max }} d \xi \mathcal{F}_{i}^{(\mathrm{DIS})}\left(\xi, Q_{D I S}^{2}\right)},
$$

where $\mathcal{F}_{i}$ denotes $F_{2}^{A}$ or $x_{A} F_{3}^{A}$ (used later for neutrino scattering). The value $Q_{D I S}^{2}$ is taken as the actual $Q^{2}$ value for a given experimental data set. For electron-carbon scattering we choose the data set [25] at $Q_{D I S}^{2}=50 \mathrm{GeV}^{2}$, because it covers most of the $\xi$ region. For a proton target [3], the integration limits for $\xi$ are conventionally chosen equal for both integrals and are defined in such a way as to cover the first and second resonance regions for each $Q^{2}$. For a free nucleon, this requirement is written as [13]

$$
\xi_{\min }^{N}=\xi\left(W=1.6 \mathrm{GeV}, Q^{2}\right), \quad \xi_{\max }^{N}=\xi\left(W=1.1 \mathrm{GeV}, Q^{2}\right),
$$

where the invariant mass for a free nucleon can be expressed in terms of $\nu$ and $Q^{2}$ as $W^{2}=(p+q)^{2}=m_{N}^{2}+2 m_{N} \nu-Q^{2}$. The upper value $W=1.6 \mathrm{GeV}$ is chosen in such a way as to cover the mass range of the four resonances taken into account, the heaviest one with the mass $M_{R}=1.535 \mathrm{GeV}$. The lower value $W=1.1 \mathrm{GeV}$ is chosen close to the pion-production threshold $W_{t h r}=1.08 \mathrm{GeV}$. In a nuclear target, the invariant mass of the struck nucleon depends on the initial momentum of the target nucleon. On the other hand, the structure functions, as well as other observables, are defined as integrals over the initial nucleon momentum. This prevents one from using $W$ in defining the integration limits. One needs an alternative variable, which can be easily determined from the lepton kinematics.

Experimentally one often (see, for example, [30]) "defines" the effective variable $\tilde{W}$ by the relation $\tilde{W}^{2}=m_{N}^{2}+$ $2 m_{N} \nu-Q^{2}$. Notice that $\tilde{W}$ is only an invariant for $\vec{p}=0$. However, it gives a reasonable feeling of the invariant mass region involved in the problem. In particular, the resonance curves presented in all figures are plotted in the region from the pion-production threshold up to $\tilde{W}=2 \mathrm{GeV}$. As was illustrated in Fig. 2 bound backward-moving nucleons allow lower $\nu$ values beyond the free-nucleon limits. Thus, as discussed at the end of Secton IIB, the threshold for the structure functions is now defined in terms of $\nu$ or $\tilde{W}$, rather than $W$. Hence, we consider two different cases in 
choosing the $\xi$ integration limits for the ratio (25). First, for a given $Q^{2}$, we choose the $\xi$ limits as in Eq. (26). That amounts to defining them by the condition

$$
\xi_{\min }=\xi\left(\tilde{W}=1.6 \mathrm{GeV}, Q^{2}\right), \quad \xi_{\max }=\xi\left(\tilde{W}=1.1 \mathrm{GeV}, Q^{2}\right)
$$

We refer to this choice as integrating "from $1.1 \mathrm{GeV}$ ". The integration limits for the DIS curve always correspond to this choice. As a second choice, for each $Q^{2}$ we integrate the resonance curve from the threshold, that is from as low $\tilde{W}$ as achievable for the nucleus under consideration. This corresponds to the threshold value at higher $\xi$ and is referred to as integrating "from threshold". With this choice we guarantee that the extended kinematical regions typical for resonance production from nuclei are taken into account. Since there is no natural threshold for the $\xi_{\min }$, for both choices it is estimated from $\tilde{W}=1.6 \mathrm{GeV}$, as defined in Eq. (27).

The results for the ratio in Eq. (25) are shown in the right panel of Fig. 4. The curve for the isoscalar free-nucleon case is the same as in Ref. [13] with the "GRV" parameterization for the DIS structure function. One can see that the carbon curve obtained by integrating "from threshold" lies above the one obtained by integrating "from $1.1 \mathrm{GeV}$ ", the difference increasing with $Q^{2}$. This indicates that the threshold region becomes more and more significant, as one can see from Fig. 3. The closer the ratio (25) gets to 1, the higher the accuracy of local duality is. Our calculations for a carbon target show that: 1) the ratio grows with $Q^{2}$, just like in the isoscalar free-nucleon case; 2) the ratio is lower than the free-nucleon value for both choices of the integration limits. This means that the integrated resonance contribution is always smaller than the integrated DIS one. In search for an explanation for this discrepancy, we

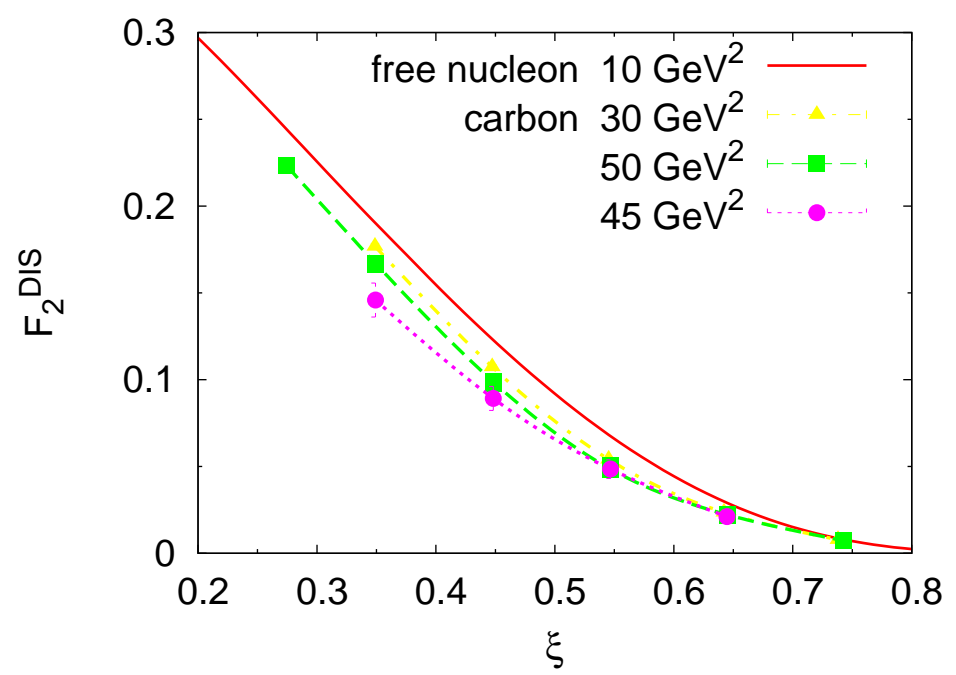

FIG. 5: Electromagnetic structure functions $F_{2}$ in DIS region for a free isoscalar nucleon as obtained via the GRV parameterization at $Q^{2}=10 \mathrm{GeV}^{2}$ (solid curve) and for a carbon nucleus as measured experimentally at $Q^{2}=30,45$ and $50 \mathrm{GeV}^{2}$.

compare how nuclear effects influence the resonance and DIS curves. As it was illlustrated in Fig. 3, the nuclear effects suppress the resonance peaks by $40 \%-50 \%$, broaden them and shift them to lower $\xi$ values. The experimental DIS values for the carbon nucleus, on the other hand, are only 5\%-10\% lower than the DIS curve for the free isoscalar nucleon. This is illustrated in Fig. 5, where the DIS structure function $F_{2}$ for a carbon target is compared to the GRV parameterization for the free isoscalar nucleon at $Q^{2}=10 \mathrm{GeV}^{2}$. In conclusion, one can say that nuclear effects have a much more dramatic effect in the resonance region than in the DIS regime. 
Similar calculations can be done for other nuclei. First of all, it would be interesting to compare an isoscalar

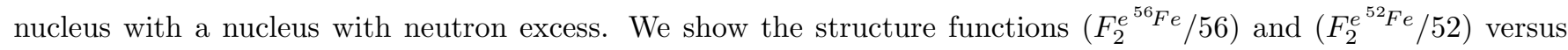
$\tilde{W}$ for several $Q^{2}$ values in the left panel of Fig. 6. The structure functions for ${ }^{52} \mathrm{Fe}$ are only marginally higher than those for ${ }^{56} \mathrm{Fe}$. This can be explained by the fact that the electromagnetic $\Delta$-production cross section is equal for proton and neutron targets. In the second resonance region, the cross sections on the proton are typically $5 \%-30 \%$ higher than those for the neutron. The overall effect, however, is hardly visible for an excess of 4 neutrons out of 56 nucleons. From an experimental point of view, it is also interesting to compare carbon with iron target nuclei. In the
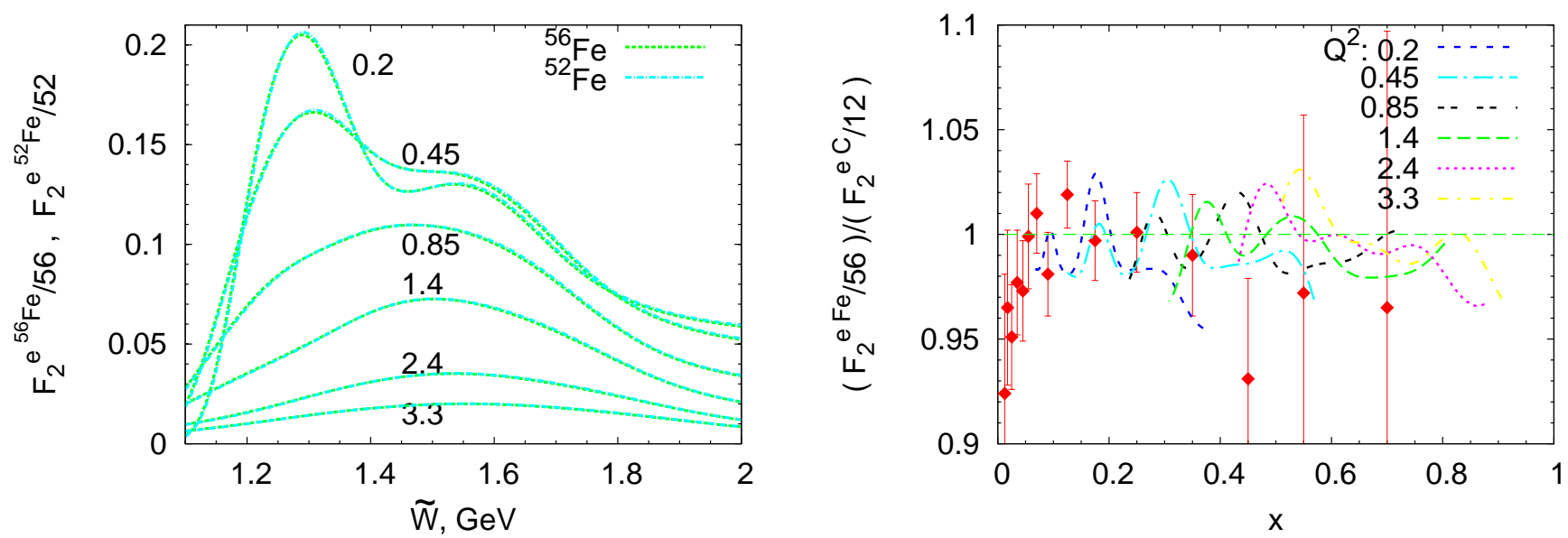

FIG. 6: (color online) (Left) Structure functions $F_{2}$ for electroproduction on iron-52 and iron-56 versus $\tilde{W}$. Curves are for $Q^{2}=0.2,0.45,0.85,1.4$, and $2.4 \mathrm{GeV}^{2}$ (indicated on the spectra). (Left) Ratio $\left(F_{2}^{e^{56} F e} / 56\right) /\left(F_{2}^{e^{12} C} / 12\right)$ versus Bjorken variable $x$ compared to DIS data 31$]$.

right panel of Fig. [6] we plot the ratio of structure functions $\left(F_{2}^{e^{56} \mathrm{Fe}} / 56\right) /\left(F_{2}^{e^{12} \mathrm{C}} / 12\right)$ versus $x$ for several values of $Q^{2}$ ranging from $0.2 \mathrm{GeV}^{2}$ to $3.3 \mathrm{GeV}^{2}$. All curves are shown in the $\xi$ region corresponding to $1.1 \mathrm{GeV}<\tilde{W}<2.0 \mathrm{GeV}$.

We stress that there is little physical meaning in the fine structure of the curves in the right panel of Fig. 6. The peaks in the curves, for example, do not coincide with the resonance peaks. As one can see, the iron structure functions appear to be very close to the carbon ones: for each $Q^{2}$ the ratio of the iron to carbon structure functions does not deviate more than $5 \%$ from the value of 1 . When averaged, this ratio slightly decreases with increasing $Q^{2}$, a behavior which is also exhibited by the DIS data presented in the same figure. The latter were measured by the NMC Collaboration [31], the mean $Q^{2}$ in the experiment varying from $20 \mathrm{GeV}^{2}$ for $x \sim 0.1$ to $60 \mathrm{GeV}^{2}$ for $x \rightarrow 1$.

\section{DUALITY IN NEUTRINOPRODUCTION}

In a previous paper [13] it was demonstrated that in neutrino reactions quark-hadron duality does not hold for proton and neutron targets separately. This is a principle feature of neutrino interactions, stemming from fundamental isospin arguments. For the charged current reaction $\nu_{\mu} p \rightarrow \mu^{-} R^{++}$, only isospin-3/2 $R^{++}$resonances are excited, in particular the $P_{33}(1232)$ resonance. Because of isospin symmetry constraints, the neutrino-proton structure functions for these resonances are three times larger than the neutrino-neutron ones. In neutrino-neutron scattering, both the isospin-3/2 resonances and the isospin- $1 / 2$ resonances contribute to the structure functions. The interplay between the resonances of different isospins allows for duality to hold with reasonable accuracy for the average over the proton 
and neutron targets. It appears reasonable that one may expect a similar picture to emerge in neutrino reactions with nuclei.
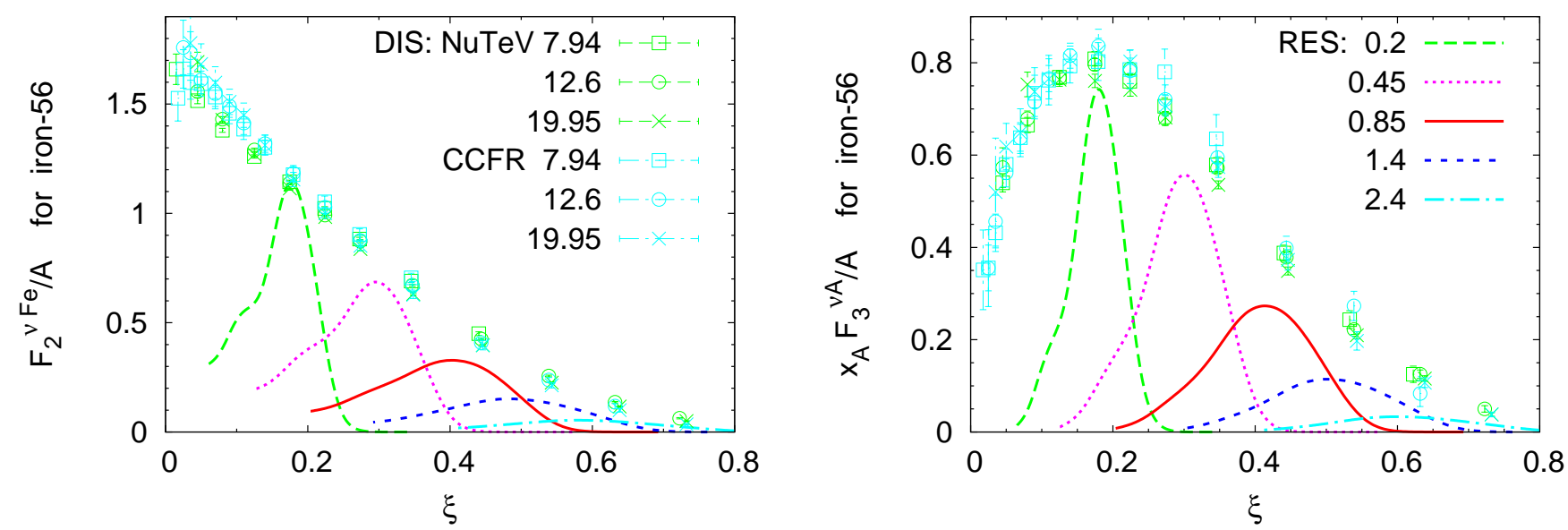

FIG. 7: (color online) The computed resonance curves $F_{2}^{\nu}{ }^{56} \mathrm{Fe} / 56$ and $x_{\mathrm{Fe}} F_{3}^{\nu{ }^{56} \mathrm{Fe}} / 56$ as a function of $\xi$, for $Q^{2}=0.2,0.45,0.85$, 1.4, and $2.4 \mathrm{GeV}^{2}$. The calculations are compared with the DIS data from Refs. [26, 27]. The DIS data refer to measurements at $Q_{D I S}^{2}=7.94,12.6$ and $19.95 \mathrm{GeV}^{2}$.
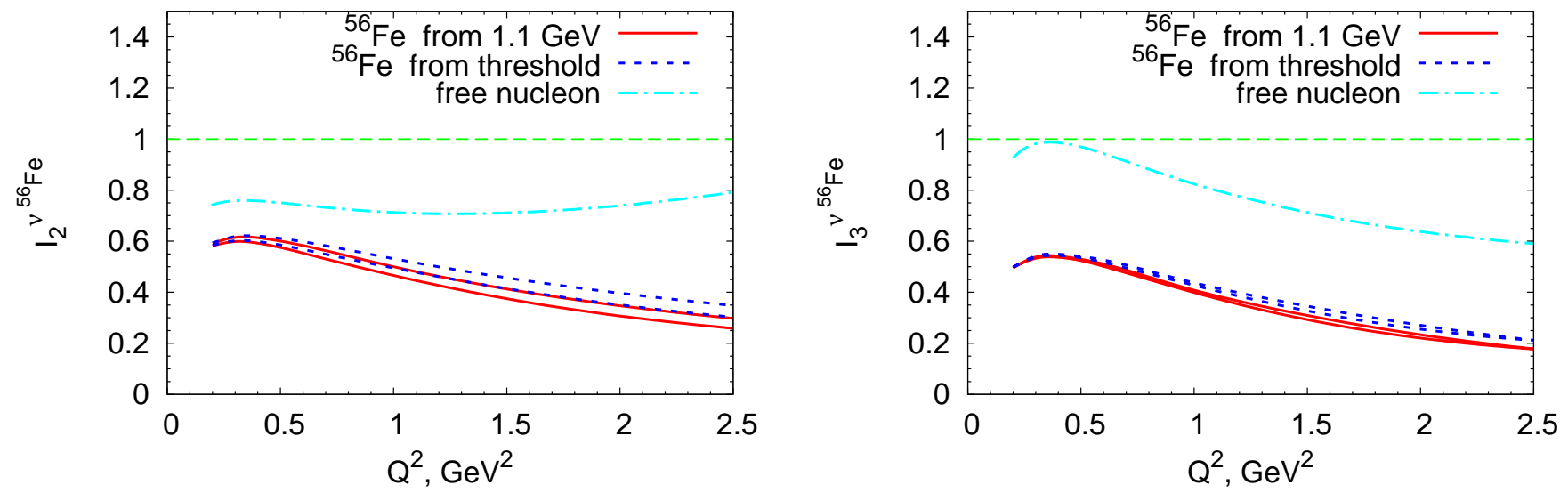

FIG. 8: (color online) Ratios $I_{2}^{\nu}{ }^{56} \mathrm{Fe}$ and $I_{3}^{\nu{ }^{56} \mathrm{Fe}}$ defined in Eq. (25) for the free nucleon (dash-dotted line) and ${ }^{56} \mathrm{Fe}$. For ${ }^{56} \mathrm{Fe}$ the results are displayed for two choices of the underlimit in the integral: $\tilde{W}=1.1 \mathrm{GeV}$ (solid line) and threshold (dotted line). For each of these two choices we have used two sets of DIS data in determining the denominator of Eq. (25). These sets of DIS data are obtained at $Q_{D I S}^{2}=12.59$ and $19.95 \mathrm{GeV}^{2}$.

The structure functions $F_{2}^{A}$ and $x_{A} F_{3}^{A}$ for neutrino-iron scattering are shown in Fig. 7 . The curves for the isoscalar free nucleon case is identical to the one presented in Ref. 13] with the "fast" fall-off of the axial form factors for the isospin- $1 / 2$ resonances.

Like for the electron-carbon results of Fig. 3, the resonance structure is hardly visible. Indeed for each $Q^{2}$ the computed resonance curves display one broad peak. The resonance structure functions are compared with the experimental data in DIS region obtained by the CCFR [26] and $\mathrm{NuTeV} \mathrm{[27]} \mathrm{collaborations.} \mathrm{It} \mathrm{appears} \mathrm{that} \mathrm{the} \mathrm{resonance}$ curves slide along the DIS curve, which indicates global duality. Like for the electron results discussed in previous section, however, the resonance $F_{2}^{A}$ and $x_{A} F_{3}^{A}$ predictions are noticeably lower than the DIS measurements. 
The ratios $I_{2}^{\nu 6} \mathrm{Fe}$ and $I_{3}^{\nu{ }^{56} \mathrm{Fe}}$ defined in Eq.(25) are shown in Fig. 8 . Our results show, that 1) these ratios are significantly smaller than $1 ; 2$ ) they are significantly smaller than the one for the free nucleon ; 3) $I_{2}$ is lower than the corresponding ratio for electroproduction; 4) $I_{2}$ and $I_{3}$ slightly decrease with $Q^{2}$ which is the opposite behavior of what was observed for electrons.
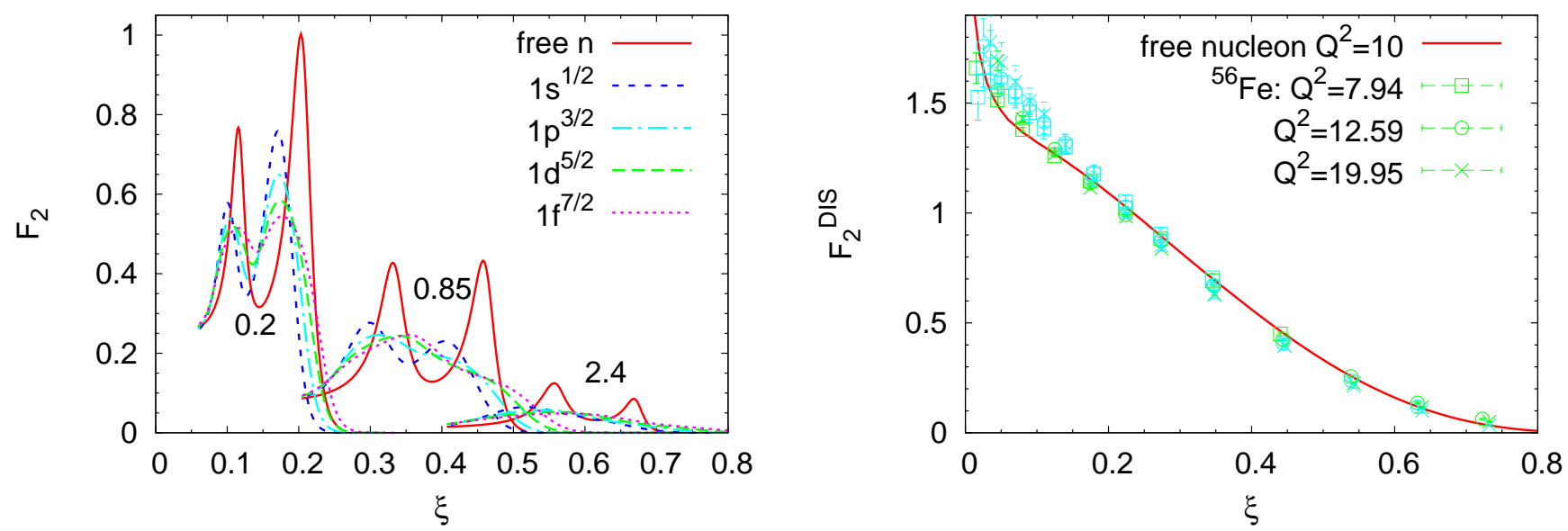

FIG. 9: (color online) (Left) Weak structure functions $F_{2}$ in the resonance region for a free neutron (solid curve), for $1 s^{1 / 2}$ (dashed curve), $1 p^{3 / 2}$ (dash-dotted curve), $1 d^{5 / 2}$ (long-dashed curve) and $1 f^{7 / 2}$ (short-dashed curve) neutrons in ${ }^{56} \mathrm{Fe}$. The three sets of curves correspond to $Q^{2}=0.2,0.85$, and $2.4 \mathrm{GeV}^{2}$. (Right) Weak structure functions $F_{2}$ in DIS region for a free isoscalar nucleon as obtained via GRV parameterization at $Q^{2}=10 \mathrm{GeV}^{2}$ (solid curve) and for iron-56 nucleus as measured experimentally [26, 27] at $Q^{2}=7.94,12.59$ and $19.95 \mathrm{GeV}^{2}$.

In an attempt to explain the above observations, we compare the free isoscalar structure functions with the ${ }^{56} \mathrm{Fe}$ ones. In the left panel of Fig. 9 the structure function $F_{2}^{A}$ for a neutron in the $1 s^{1 / 2}, 1 p^{3 / 2}, 1 d^{5 / 2}$ and $1 f^{7 / 2}$ iron shells are contrasted with the structure function for a free neutron. For a fixed $Q^{2}$, the peak at the larger value of the Nachtmann variable corresponds to the $\Delta$ resonance. The peak at smaller $\xi$ corresponds to the second resonance region. It is clear that the nuclear effects reduce the peaks by about $30-50 \%$ and shift them to lower $\xi$ values in comparison with the free nucleon case. The suppression is most significant for the single-particle shells close to the Fermi surface.

In close resemblance to what was observed in the discussion of the electron-nucleus cross sections of previous section, the peculiar Fermi smearing pattern for each shell introduces additional averaging when summing over shells. For a bound proton, the effect of suppression is nearly the same. In the right panel of Fig. 9 measured DIS structure functions for ${ }^{56} \mathrm{Fe}$ at various $Q^{2}$ are compared to the GRV parameterization $\left(Q^{2}=10 \mathrm{GeV}^{2}\right)$ for a free isoscalar nucleon [13]. It is obvious that the measured nuclear DIS structure functions are very similar to the free-nucleon ones. Thus, we predict a substantial nuclear reduction of the resonance strength, whereas the data in the DIS region do not point to such a reduction. This explains the computed low values of the ratios in Fig. 8

We wish to stress that the low values of $I_{2}^{\nu{ }^{56} \mathrm{Fe}}$ and $I_{3}^{\nu{ }^{56} \mathrm{Fe}}$ are not related to the neutron excess. We remind that the neutron structure functions for the $\Delta$ resonance are 3 times smaller than the proton ones. The structure function for isoscalar ${ }^{52} \mathrm{Fe}$ is only about $5 \%$ larger than for ${ }^{56} \mathrm{Fe}$. This is shown in Fig. 10 for $F_{2}$ and $x_{A} F_{3}$. The effect can be easily estimated from $(26 \cdot 3 \cdot f+30 \cdot f) /(26 \cdot 3 \cdot f+26 \cdot f) \approx 1.04$, where $f$ is the neutron structure function in the $\Delta$ region. In the second resonance region the difference is even smaller. 

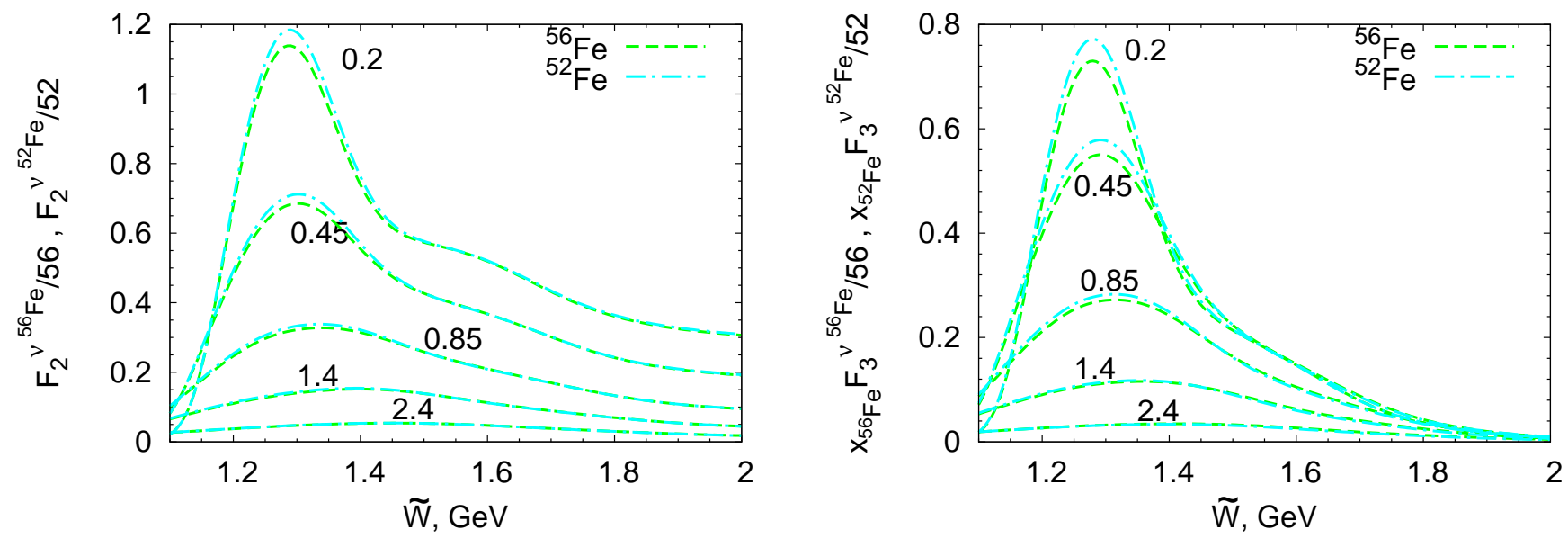

FIG. 10: (color online) Structure functions $F_{2}^{A}$ (left) and $x_{A} F_{3}^{A} / A$ (right) for neutrinoproduction on iron-52 and iron-56 versus $\tilde{W}$. Curves in the resonance region are for $Q^{2}=0.2,0.45,0.85,1.4$, and $2.4 \mathrm{GeV}^{2}$ (indicated on the spectra).
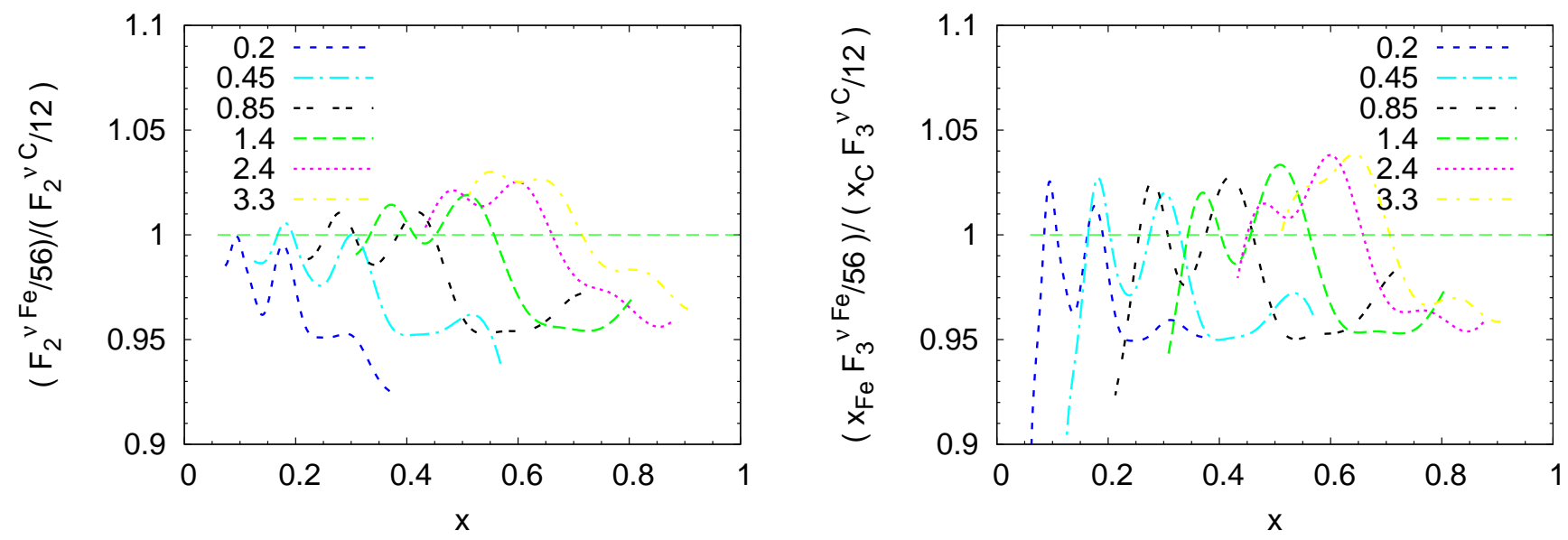

FIG. 11: (color online) Ratios $\left(F_{2}^{\nu^{56} \mathrm{Fe}} / 56\right) /\left(F_{2}^{\nu^{12} C} / 12\right)$ (left) and $\left(x_{56} \mathrm{Fe} F_{3}^{\left.\nu^{56} \mathrm{Fe} / 56\right)} /\left(x_{52} \mathrm{Fe} F_{3}^{\nu^{12} C} / 12\right)\right.$ (right) versus Bjorken variable $x$ for $Q^{2}=0.2,0.45,0.85,1.4,2.4$ and $3.3 \mathrm{GeV}^{2}$

It is also interesting to make a comparison with the carbon nucleus. The ratios of iron to carbon structure functions $F_{2}^{A}$ and $x_{A} F_{3}^{A}$ versus $x$ are shown in Fig. 11. For each $Q^{2}$ the $\xi$ range corresponds to $1.1<\tilde{W}<2.0 \mathrm{GeV}$. Like in the case of electromagnetic reaction, the ratios are close to 1, but a bit lower in general and the average is slightly increasing with $Q^{2}$. Remark that the peaks in Fig. 11 are not related to resonances and that the fluctuations which are of the order of $5 \%$ can be attributed to subtleties in the shell structure of the various target nuclei.

\section{SUMMARY}

In view of the current experimental activities, there is great need for an efficient framework for reliably predicting neutrino-nucleus cross sections and for a deeper understanding of quark-hadron duality in nuclei. We performed a phenomenological study of duality in electron-nucleus and neutrino-nucleus structure functions.

Using the Dortmund-group model for the production of the first four lowest-lying nucleon resonances and using single-particle wavefunctions from the Hartree approximation to the relativistic $\sigma \omega$ model, we computed the structure 
functions $x_{A} F_{1}^{A}, F_{2}^{A}$ and $x_{A} F_{3}^{A}$ in the resonance region for carbon and iron targets and compared them with the measured DIS ones. At the same time we compared the computed resonance structure functions for nuclei with those for a free nucleon. For quantitative comparisons, we defined the ratios $I_{i}\left(Q^{2}\right)$ of integrated resonance to DIS structure functions. Perfect quark-hadron duality is reached for $I_{i}\left(Q^{2}\right)$ values of unity.

Summarizing our results, we observe that the computed resonance contribution to the lepton-nucleus structure functions is qualitatively consistent with the measured DIS structure functions. This means that global quark-hadron duality holds for nuclei. The computed integrated resonance strength, however, is about half of the measured DIS one. Contrary to the free nucleon case, where the ratios $I_{i}\left(Q^{2}\right)$ are at the level of 0.8 , we find for nuclei 0.6 for electroproduction and 0.4 for neutrinoproduction. This points towards a scale dependence in the role of the nuclear effects. It is obvious that nuclear effects act differently at lower $Q^{2}$ (resonance regime) than at higher $Q^{2}$ (DIS regime).

In our presented analysis we include the resonance contributions and ignored the role of the background terms. Further investigations require a theoretical or phenomenological model for the background contributions in the first and second resonance region. One could for example estimate the role of the background contribution to the $\Delta^{-}$ resonance region within the context of the non-linear sigma model [32]. Extending these or similar models to higher $W$ values and incorporating them in a model for lepton reactions with nuclei could be the next step in exploring quark-hadron duality.

\section{Acknowledgments}

The authors acknowledge financial support from the Research Foundation - Flanders (FWO), and the Research Council of Ghent University.

[1] E. D. Bloom and F. J. Gilman, Phys. Rev. Lett. 25, 1140 (1970).

[2] W. Melnitchouk, R. Ent, and C. Keppel, Phys. Rept. 406, 127 (2005), hep-ph/0501217.

[3] I. Niculescu et al., Phys. Rev. Lett. 85, 1186 (2000).

[4] J. Arrington, R. Ent, C. E. Keppel, J. Mammei, and I. Niculescu, Phys. Rev. C73, 035205 (2006), nucl-ex/0307012.

[5] D. Boehnlein (MINERvA), AIP Conf. Proc. 967, 304 (2007).

[6] C. J. Solano Salinas, A. Chamorro, and C. Romero (MINERvA), AIP Conf. Proc. 947, 239 (2007).

[7] Minerva collaboration homepage, URL http://minerva.fnal.gov.

[8] J. L. Alcaraz-Aunion and J. Catala-Perez, AIP Conf. Proc. 967, 307 (2007).

[9] K. Hiraide (SciBooNE), AIP Conf. Proc. 967, 316 (2007).

[10] Sciboone collaboration homepage, URL http://www-sciboone.fnal.gov

[11] K. Matsui, T. Sato, and T. S. H. Lee, Phys. Rev. C72, 025204 (2005), nucl-th/0504051.

[12] K. M. Graczyk, C. Juszczak, and J. T. Sobczyk, Nucl. Phys. A781, 227 (2007), hep-ph/0512015.

[13] O. Lalakulich, W. Melnitchouk, and E. A. Paschos, Phys. Rev. C75, 015202 (2007), hep-ph/0608058.

[14] B. D. Serot and J. D. Walecka, Adv. Nucl. Phys. 16, 1 (1986).

[15] R. J. Furnstahl, B. D. Serot, and H.-B. Tang, Nucl. Phys. A615, 441 (1997), nucl-th/9608035.

[16] M. C. Martinez et al., Phys. Rev. C73, 024607 (2006), nucl-th/0505008.

[17] O. Benhar and D. Meloni, Nucl. Phys. A789, 379 (2007), hep-ph/0610403. 
[18] T. Sato, D. Uno, and T. S. H. Lee, Phys. Rev. C67, 065201 (2003), nucl-th/0303050.

[19] D. Rein and L. M. Sehgal, Ann. Phys. 133, 79 (1981).

[20] O. Lalakulich, E. A. Paschos, and G. Piranishvili, Phys. Rev. D74, 014009 (2006), hep-ph/0602210.

[21] T. C. Ferree and D. S. Koltun, Phys. Rev. C55, 253 (1997), nucl-th/9512021.

[22] G. Vereshkov and N. Volchanskiy, Phys. Rev. D76, 073007 (2007), 0707.0051.

[23] W. B. Atwood and G. B. West, Phys. Rev. D7, 773 (1973).

[24] D. Bollini et al., Phys. Lett. B104, 403 (1981).

[25] A. C. Benvenuti et al. (BCDMS), Phys. Lett. B195, 91 (1987).

[26] W. G. Seligman et al., Phys. Rev. Lett. 79, 1213 (1997).

[27] M. Tzanov et al. (NuTeV), Phys. Rev. D74, 012008 (2006), hep-ex/0509010.

[28] O. Nachtmann, Nucl. Phys. B63, 237 (1973).

[29] H. Georgi and H. D. Politzer, Phys. Rev. D14, 1829 (1976).

[30] R. M. Sealock et al., Phys. Rev. Lett. 62, 1350 (1989).

[31] M. Arneodo et al. (New Muon), Nucl. Phys. B481, 3 (1996).

[32] E. Hernandez, J. Nieves, and M. Valverde, Phys. Rev. D76, 033005 (2007), hep-ph/0701149. 